\title{
HIGROSCOPICIDADE DAS SEMENTES DE SORGO-SACARINO ${ }^{1}$
}

Doi:http://dx.doi.org/10.1590/1809-4430-Eng.Agric.v36n3p 515-524/2016

\section{RENAN ULLMANN ${ }^{2}$, OSVALDO RESENDE ${ }^{3}$, DANIEL E. C. OLIVEIRA ${ }^{4}$, LÍLIAN M. COSTA ${ }^{5}$, TARCÍSIO H. CHAVES ${ }^{6}$}

\begin{abstract}
RESUMO: Objetivou-se, neste trabalho, determinar as isotermas de dessorção das sementes de sorgo-sacarino para diversas condições de temperatura e atividades de água e obter o calor isostérico de dessorção. As sementes foram submetidas à secagem em estufa com ventilação de ar forçada a $40{ }^{\circ} \mathrm{C}$ até atingir os teores de água de aproximadamente $24 ; 19 ; 15 ; 12$ e 8 (\% b.s.). As isotermas de dessorção das sementes de sorgo-sacarino foram obtidas pelo método estático indireto, sendo a atividade de água $\left(\mathrm{a}_{\mathrm{w}}\right)$ determinada por meio do equipamento Hygropalm Model Aw 1. Para o controle da temperatura, utilizou-se de B.O.D., regulada a 10; 20; 30 e $40{ }^{\circ} \mathrm{C}$. Aos dados experimentais, foram ajustados modelos matemáticos frequentemente utilizados para a representação da higroscopicidade de produtos vegetais. Verificou-se que o modelo de Chung-pfost é o que melhor representa a higroscopicidade das sementes de sorgo-sacarino. O calor isostérico integral de dessorção aumenta com a redução do teor de água, sendo que os valores, para as sementes de sorgo-sacarino na faixa de teor de água de 7,9 a 28,0 (\% b.s.), variam de 3.136,54 a $2.493,33 \mathrm{~kJ} \cdot \mathrm{kg}^{-1}$.
\end{abstract}

PALAVRAS-CHAVE: modelagem, calor isostérico, isotermas.

\section{HYGROSCOPICITY OF SWEET SORGHUM SEEDS}

ABSTRACT: This study focused in determining the sorption isotherms of sweet sorghum seeds at varied conditions of temperature and water activity, ascertaining the isosteric heat of desorption. Seeds were dried in a forced-air oven at $40{ }^{\circ} \mathrm{C}$ up to reach moisture levels of $24,19,15,12$ and $8 \%$ (d.b.). Desorption isotherms of sorghum seeds were estimated by the indirect static method, and water activity (aw) was determined using a Hygropalm aw model 1 device. Temperature control was set at $10,20,30$, and $40{ }^{\circ} \mathrm{C}$ in the B.O.D chambers. Experimental data were fitted using mathematical models regularly used to represent hygroscopicity of plant products. Results showed Chung-Pfost model as the one that best represents sorghum seed hygroscopicity. The isosteric heat of sorption increases with decreasing moisture contents; and the values for sorghum seeds with

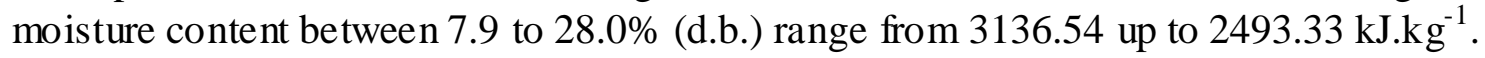

KEY WORDS: modeling, isosteric heat, isotherms.

\section{INTRODUÇÃO}

Diversas plantas vêm sendo estudadas como fontes de energia renovável para diversificar a matriz energética; dentre estas, o sorgo-sacarino está surgindo como potencial matéria-prima e em complemento à cultura da cana-de-açúcar, para a produção de etanol e bagaço (TEIXEIRA et al., 1997).

Dentre esses estudos, os relacionados à pós-colheita são de extrema importância para garantir a manutenção da qualidade dos produtos vegetais colhidos. Dentro dessas etapas, a secagem e o armazenamento destacam-se como uma das principais (ULLMANN et al., 2010).

\footnotetext{
${ }^{1}$ Parte da dissertação de mestrado do primeiro autor.

${ }^{2}$ Eng $^{\circ}$ A grônomo, Mestre, IF Goiano. Rio Verde, GO, renanullmann @ hotmail.com

${ }^{3}$ Eng $^{\circ}$ A grícola, Prof. Doutor, IF Goiano. Rio Verde, GO, osvresende@y ahoo.com.br

${ }^{4}$ Eng $^{\circ}$ A grícola, Prof. Doutor, IF Goiano. Rio Verde, GO, oliv eira.d.e.c@ gmail.com

${ }^{5}$ Tecnóloga em Produção de Grãos, Mestre, IF Goiano. Rio Verde, GO, lilian.2207@ hotmail.com

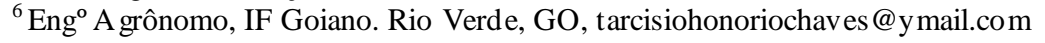


No desenvolvimento de equipamentos utilizados para a secagem de sementes, são de fundamental importância a simulação e a obtenção de informações teóricas a respeito do comportamento de cada produto durante a remoção de água (RESENDE et al., 2011).

Dessa maneira, torna-se necessário o conhecimento das relações existentes entre as sementes, a temperatura, a umidade relativa do ar e o teor de água em que se encontra o produto, visando a amenizar as variações que podem vir a deteriorar o material.

Assim, a simulação matemática permite fazer a otimização desses processos em tempo significativamente menor e de maneira econômica (DALPASQUALE \& SPERANDIO, 2010).

O estudo das isotermas é necessário para conhecer-se a relação com as características físicas, químicas e a estabilidade dos produtos desidratados ou parcialmente desidratados (HUBINGER et al., 2009).

Segundo HENAO et al. (2009), as isotermas de sorção são usadas para vários propósitos, como determinação do tempo de secagem e geração de informações referentes ao armazenamento adequado e à manutenção da qualidade dos produtos.

O comportamento higroscópico de diversos produtos agrícolas tem sido estudado por meio de modelos matemáticos empíricos, uma vez que nenhum modelo teórico desenvolvido tem sido capaz de predizer, com precisão, o teor de água de equilíbrio para uma ampla faixa de temperatura e de atividade (FARAHNAKY et al., 2009; HENAO et al., 2009; CHEN \& WENG, 2010).

Diversos trabalhos têm sido realizados recentemente com a finalidade de estudar a higroscopicidade de diferentes produtos vegetais, tais como pimenta-malagueta (SILVA \& RODOVALHO, 2012), crambe (COSTA et al., 2013), pinhão-manso (SANTOS et al., 2013), caju (CAETANO et al., 2012), quiabo (GONELI et al., 2010), nabo-forrageiro (SOUSA et al., 2013) e milho (SMANIOTTO et al., 2012).

Segundo Lima et al. (2008), o calor isostérico é essencial nos estudos de secagem e armazenamento de produtos agrícolas servindo de bom parâmetro para estimar-se a quantidade mínima de calor requerida para remover uma quantidade de água.

Diante da importância do conhecimento e do estudo da higroscopicidade dos produtos agrícolas, nota-se que, na literatura, existe limitação de estudos relacionados às sementes de sorgo sacarino. Assim, o objetivo neste trabalho foi determinar as isotermas de sorção das sementes de sorgo-sacarino para diversas condições de temperatura e de atividades de água e obter o calor isostérico de dessorção.

\section{MATERIAL E MÉTODOS}

O trabalho foi desenvolvido nos Laboratórios de Pós-Colheita de Produtos Vegetais e no Laboratório de Sementes do Instituto Federal de Educação, Ciência e Tecnologia Goiano (IF Goiano - Campus Rio Verde), situados no município de Rio Verde-GO.

As sementes de sorgo-sacarino foram colhidas manualmente depois da maturação, com teor de água de aproximadamente $28 \%$ (b.s.), e posteriormente foram encaminhadas para o processo de secagem em estufa com ventilação de ar forçada a $40{ }^{\circ} \mathrm{C}$ até atingir os teores de água de aproximadamente $24 ; 19 ; 15 ; 12$ e $8 \%$ (b.s.).

Para a obtenção dos dados para a modelagem da dessorção das sementes de sorgo-sacarino, utilizou-se do método estático indireto, no qual 12 gramas de produto nos teores de água de 24; 19; 15; 12 e 8 (\% b.s.) foram inseridos dentro de câmara isolada, e foi determinada a atividade de água $\left(a_{\mathrm{w}}\right)$ por meio do equipamento Hygropalm Model Aw. No método estático indireto, utilizam-se valores preestabelecidos de teores de água do produto e obtém-se a $\mathrm{a}_{\mathrm{w}}$ em condições controladas de temperatura. Para o controle da temperatura, utilizou-se de uma câmara do tipo B.O.D., regulada nas temperaturas de $10 ; 20 ; 30$ e $40{ }^{\circ} \mathrm{C}$, e as leituras da $\mathrm{a}_{\mathrm{w}}$ foram realizadas quando a temperatura das amostras se estabilizava. 
Os teores de água das sementes de sorgo-sacarino foram determinados por gravimetria, utilizando uma estufa de secagem regulada com temperatura do ar em $105 \pm 1{ }^{\circ} \mathrm{C}$, durante 24 horas, em três repetições, segundo BRASIL (2009), com adaptações.

Aos dados experimentais foram ajustados modelos matemáticos que são frequentemente utilizados para a representação da higroscopicidade de produtos vegetais, conforme Tabela 1.

TABELA 1. Modelos matemáticos utilizados para predizer a higroscopicidade de produtos vegetais. Mathematical models used to predict hygroscopicity of plant products.

\begin{tabular}{|c|c|c|c|}
\hline Designação do modelo & $\mathrm{Mo}$ & elo & \\
\hline $\mathrm{Xe}^{*}=\exp \left\{\mathrm{a}-(\mathrm{b} \cdot \mathrm{T})+\left[\mathrm{c} \cdot \exp \left(\mathrm{a}_{\mathrm{w}}\right)\right]\right\}$ & Sigma Copace & Corrêa et al. (1995) & $(1)$ \\
\hline $\mathrm{Xe} \mathrm{e}^{*}=(\mathrm{a}+\mathrm{b} \cdot \mathrm{T}) /\left[\left(1-\mathrm{a}_{\mathrm{w}}\right) / \mathrm{a}_{\mathrm{w}}\right]^{\frac{1}{\mathrm{c}}}$ & Oswin Modificado & $\begin{array}{l}\text { Iguaz e Vírseda } \\
\text { (2007) }\end{array}$ & (2) \\
\hline $\mathrm{Xe}^{*}=\left[\ln \left(1-\mathrm{a}_{\mathrm{w}}\right) /(-\mathrm{a} \cdot(\mathrm{T}+\mathrm{b}))\right]^{\frac{1}{\mathrm{c}}}$ & $\begin{array}{l}\text { Henderson } \\
\text { Modificado }\end{array}$ & Henderson (1952) & (3) \\
\hline $\mathrm{Xe}^{*}=\exp \left[\mathrm{a}-(\mathrm{b} \cdot \mathrm{T})+\left(\mathrm{c} \cdot \mathrm{a}_{\mathrm{w}}\right)\right]$ & Copace & Corrêa et al. (1995) & (4) \\
\hline $\mathrm{Xe}^{*}=\mathrm{a}-\mathrm{b} \cdot \ln \left[-(\mathrm{T}+\mathrm{c}) \cdot \ln \left(\mathrm{a}_{\mathrm{w}}\right)\right]$ & Chung-Pfost & $\begin{array}{l}\text { Chung e Pfost et al. } \\
\text { (1967) }\end{array}$ & $(5)$ \\
\hline $\mathrm{Xe} \mathrm{e}^{*}=\left[\exp (\mathrm{a}-\mathrm{b} \cdot \mathrm{T}) /-\ln \left(\mathrm{a}_{\mathrm{w}}\right)\right]^{\frac{1}{\mathrm{c}}}$ & Halsey Modificado & Halsey (1948) & (6) \\
\hline $\mathrm{Xe}^{*}=\left[\ln \left(1-\mathrm{a}_{\mathrm{w}}\right) /(-\mathrm{a} \cdot(\mathrm{T}+273,16))\right]^{\frac{1}{\mathrm{~b}}}$ & Henderson & Henderson (1952) & (7) \\
\hline $\mathrm{Xe}^{*}=\mathrm{a} \cdot\left(\frac{\mathrm{a}_{\mathrm{w}}^{\mathrm{b}}}{\mathrm{T}^{\mathrm{c}}}\right)$ & Sabbah & $\operatorname{ASAE}(2003)$ & (8) \\
\hline$X e^{*}=\left(a \cdot b \cdot c \cdot a_{w}\right) /\left[\left(1-c \cdot a_{w}\right) \cdot\left(1-c \cdot a_{w}+b \cdot c \cdot a_{w}\right)\right]$ & GAB & $\begin{array}{c}\text { Van Der BERG } \\
(1984)\end{array}$ & (9) \\
\hline $\mathrm{Xe}^{*}=\left[\log \left(1-\mathrm{a}_{\mathrm{w}}\right) /\left(\mathrm{a} \cdot\left(\mathrm{T}^{\mathrm{b}}\right)\right)\right]^{\frac{1}{\mathrm{c}}}$ & Cavalcanti Mata & $\begin{array}{l}\text { Cavalcanti Mata et } \\
\text { al. (2003) }\end{array}$ & $(10)$ \\
\hline $\mathrm{Xe}^{*}=\left\{1 /\left[\left(1-\mathrm{a}_{\mathrm{w}}\right) \cdot(1 / \mathrm{a} \cdot \mathrm{b}+((\mathrm{a}-1) / \mathrm{a} \cdot \mathrm{b}))\right]\right\}$ & BET & $\begin{array}{l}\text { Park \& Nogueira } \\
\text { (1992) }\end{array}$ & (11) \\
\hline $\mathrm{Xe}^{*}=(\mathrm{a}+\mathrm{bT}) /\left[\left(1-\mathrm{a}_{\mathrm{w}}\right) / \mathrm{a}_{\mathrm{w}}\right]^{1 / \mathrm{c}}$ & Oswin & $\begin{array}{c}\text { Chinnan \& } \\
\text { Beachaut (1985) }\end{array}$ & (12) \\
\hline
\end{tabular}

em que,

$\mathrm{Xe}^{*}$ : teor de água de equilíbrio, \% b.s.;

$\mathrm{a}_{\mathrm{w}}$ : atividade de água, decimal;

T: temperatura, ${ }^{\circ} \mathrm{C}$, e

a, b, c: coeficientes de ajuste dos modelos que depende $m$ do produto.

Os modelos matemáticos foram ajustados aos dados experimentais por meio de análise de regressão não linear pelo método Gauss-Newton. Os modelos foram selecionados considerando a magnitude do coeficiente de determinação $\left(\mathrm{R}^{2}\right)$, o erro médio relativo $(\mathrm{P})$ e o erro médio estimado (SE).

$$
\begin{aligned}
& P=\frac{100}{N} \sum \frac{|\mathrm{Y}-\hat{\mathrm{Y}}|}{\mathrm{Y}} \\
& \mathrm{SE}=\sqrt{\frac{\sum(\mathrm{Y}-\hat{\mathrm{Y}})^{2}}{\mathrm{GLR}}}
\end{aligned}
$$


em que,

Y: valor experimental;

$\hat{\mathrm{Y}}$ : valor estimado pelo modelo;

$\mathrm{N}$ : número de observações experimentais, e

GLR: graus de liberdade do modelo (número de observações experimentais menos o número de coeficientes do modelo).

Para obter-se o calor isostérico de dessorção, os valores de atividade de água, temperatura e teor de água de equilíbrio foram obtidos a partir das isotermas de dessorção das sementes de sorgosacarino, utilizando o modelo que melhor se ajustou aos dados experimentais.

Para os cálculos do calor isostérico líquido de sorção, para cada teor de água de equilíbrio, utilizou-se da equação de Clausius-Clayperon (IGLESIAS \& CHIRIFE, 1976):

$$
\frac{\partial \operatorname{In}\left(\mathrm{a}_{\mathrm{w}}\right)}{\partial \mathrm{T}}=\frac{\Delta \mathrm{h}_{\mathrm{st}}}{\mathrm{RT}_{\mathrm{a}}^{2}}
$$

em que,

$\mathrm{a}_{\mathrm{w}}$ : atividade de água, decimal;

$\mathrm{T}_{\mathrm{a}}$ : temperatura absoluta, $\mathrm{K}$;

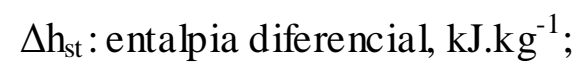

$\mathrm{R}$ : constante universaldos gases, $0,287 \mathrm{~kJ} \cdot \mathrm{kg}^{-1} \cdot \mathrm{K}^{-1}$ sendo para o vapor d'água $=0,4619 \mathrm{~kJ} \cdot \mathrm{kg}^{-1} \cdot \mathrm{K}^{-1}$.

Integrando a [eq. (15)] e assumindo que o calor isostérico líquido de sorção seja independente da temperatura, o calor isostérico líquido de sorção, para cada teor de água de equilíbrio, foi obtido conforme a [eq. (16)] (WANG \& BRENNAN, 1991):

$$
\operatorname{In}\left(\mathrm{a}_{\mathrm{w}}\right)=-\left(\frac{\Delta \mathrm{h}_{\mathrm{st}}}{\mathrm{R}}\right) \cdot \frac{1}{\mathrm{~T}_{\mathrm{a}}}+\mathrm{C}
$$

em que,

C: constante de integração.

O calor isos térico integral de sorção foi obtido adicionando-se aos valores de calor isostérico líquido de sorção (Equação 16) o valor do calor latente de vaporização da água livre, de acordo com a [eq. (17)].

$$
\mathrm{Q}_{\mathrm{st}}=\Delta \mathrm{h}_{\mathrm{st}}+\mathrm{L}=\mathrm{a} \cdot \exp \left(-\mathrm{b} \cdot \mathrm{X \textrm {e } ^ { * }}\right)+\mathrm{L}
$$

em que,

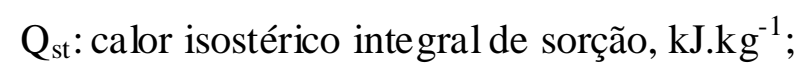

L: calor latente de vaporização da água livre, $\mathrm{kJ}_{\mathrm{kg}}{ }^{-1}$;

$\mathrm{Xe}^{*}$ : teor de água de equilíbrio, \% b.s., e

a, b: coeficientes do modelo.

$\mathrm{O}$ calor latente de vaporização da água livre $\left(\mathrm{kJ} \cdot \mathrm{kg}^{-1}\right)$, à temperatura de equilíbrio $\left({ }^{\circ} \mathrm{C}\right)$, foi calculado, utilizando a temperatura média ( T) na faixa em estudo, em ${ }^{\circ} \mathrm{C}$, por meio da seguinte equação:

$$
\mathrm{L}=2502,2-2,39 \cdot \mathrm{T}
$$




\section{RESULTADOS E DISCUSSÃO}

Na Tabela 2, estão apresentados o coeficiente de determinação $\left(\mathrm{R}^{2}\right)$, os valores do erro médio relativo, erro médio estimado e os coeficientes para os modelos de higroscopicidade ajustados as sementes de sorgo-sacarino, obtidos por dessorção, para diferentes condições de temperatura e atividades de água.

TABELA 2. Parâmetros dos modelos de equilíbrio higroscópico para as sementes de sorgosacarino, com seus respectivos erros médios estimado (SE) e relativo (P), teste de qui-quadrado $\left(\chi^{2}\right)$, coeficientes de determinação $\left(\mathrm{R}^{2}\right)$. Parameters of hygroscopic equilibrium models for $s$ weet sorghum seeds and their respective estimated (SE) and relative (P) mean errors, as well as coefficients of determination $\left(R^{2}\right)$.

\begin{tabular}{ccccccc}
\hline Modelos & SE & $\mathrm{P}$ & $\mathrm{R}^{2}$ & $\mathrm{a}$ & $\mathrm{b}$ & $\mathrm{c}$ \\
\hline Chung-pfost & 1,31 & 7,67 & 96,65 & 39,11505 & 39,11505 & 39,11505 \\
Copace & 1,59 & 8,72 & 95,06 & 1,416670 & 1,416670 & 1,416670 \\
GAB & 2,08 & 11,16 & 91,55 & - & - & - \\
Halsey Modificado & 1,42 & 9,25 & 96,09 & 5,663230 & 5,663230 & 5,663230 \\
Sabbah & 1,82 & 8,38 & 93,56 & 43,40769 & 43,40769 & 43,40769 \\
Sigma Copace & 1,53 & 8,99 & 95,45 & 0,7741 & 0,7741 & 0,7741 \\
Oswin Modificado & 1,31 & 8,52 & 96,66 & 13,06808 & 13,06808 & 13,06808 \\
Cavalcanti Mata & 1,43 & 8,08 & 96,04 & 0,013627 & 0,013627 & 0,013627 \\
Henderson Modificado & 1,35 & 7,91 & 96,44 & 0,00025 & 0,00025 & 0,00025 \\
Henderson & 1,65 & 9,33 & 94,41 & 0,000084 & 0,000084 & - \\
BET & 6,39 & 36,29 & 15,82 & - & - & - \\
Oswin & 1,31 & 8,52 & 96,66 & 13,06809 & 13,06809 & 13,06809 \\
\hline
\end{tabular}

Com relação ao erro médio estimado ( $\mathrm{SE}$ ), os modelos apresentaram baixos valores, com exceção de BET e GAB, que apresentaram as maiores magnitudes. De acordo com DRAPER \& SMITH (1998), a capacidade de um modelo representar adequadamente um determinado processo é inversamente proporcional ao valor do erro médio estimado.

Analisando os valores de erro médio relativo (P), verifica-se que, exceto os modelos de GAB e BET, os demais apresentaram magnitudes inferiores a 10\% para os modelos estudados.

$\mathrm{O}$ coeficiente de determinação $\left(\mathrm{R}^{2}\right)$ apresentou, com exceção de BET, valores superiores a 91,5\%, no qual, segundo MADAMBA et al. (1996), expressa uma representação satisfatória do processo em estudo; porém estes pesquisadores afirmam que a utilização do coeficiente de determinação, isoladamente para a seleção dos modelos não lineares, não constitui um bom parâmetro de representação.

Desta forma, com exceção dos modelos de BET e GAB, todos os demais podem ser usados para representar a higroscopicidade da espécie avaliada; sendo assim, o modelo de Chung-pfost, que vem sendo usado tradicionalmente para produtos amiláceos, foi selecionado para representar a higroscopicidade das sementes de sorgo-sacarino.

Na Figura 1, estão apresentados os valores experimentais do teor de água de equilíbrio das sementes de sorgo sacarino, obtidos por dessorção, bem como as isotermas de dessorção estimadas pelo modelo de Chung-pfost para as temperaturas de $10 ; 20 ; 30$ e $40{ }^{\circ} \mathrm{C}$. 


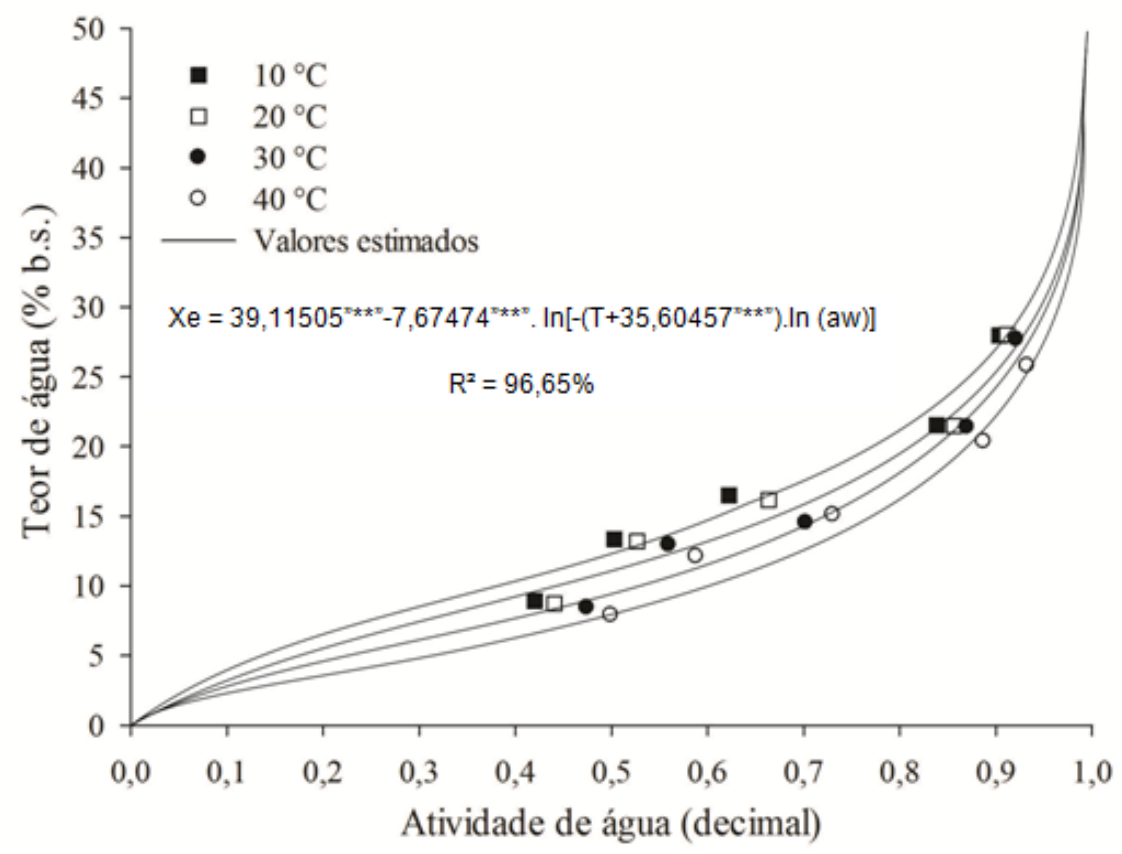

** Significativo a $5 \%$, pelo teste $\mathrm{t}$

FIGURA 1. Valores experimentais da atividade de água e isotermas de dessorção estimadas pelo modelo de Chung-pfost para as sementes de sorgo-sacarino em diferentes condições de temperatura e atividades de água. Water activity and sorption isotherms estimated by the Chung-Pfost model for sweet sorghum seeds at different temperatures and water activities.

Verificou-se que, para a atividade de água constante, o aumento da temperatura promove a redução do teor de água de equilíbrio, seguindo a tendência da maioria dos produtos agrícolas (HUBINGER et al. 2009).

Diferentes pesquisadores também verificaram que o modelo de Chung-pfost foi o que melhor se ajustou aos seus dados experimentais, como sementes de quiabo (GONELI et al., 2010) e caju-de-árvore-do-cerrado (CAETANO et al., 2012).

Diante da representação satisfatória do equilíbrio higroscópico das sementes de sorgosacarino, o modelo de Chung-pfost foi utilizado para o cálculo dos valores de atividade de água, necessários para a determinação dos valores do calor isostérico líquido de dessorção. Observou-se que, com aumento do teor de água e da temperatura, ocorreu acréscimo da atividade da água.

Na Figura 2, são apresentadas as curvas do logaritmo neperiano da atividade da água para específicos valores de teor de água de equilíbrio (\% b.s.), em função do inverso da temperatura absoluta $(\mathrm{K})$, para as sementes de sorgo-sacarino. 


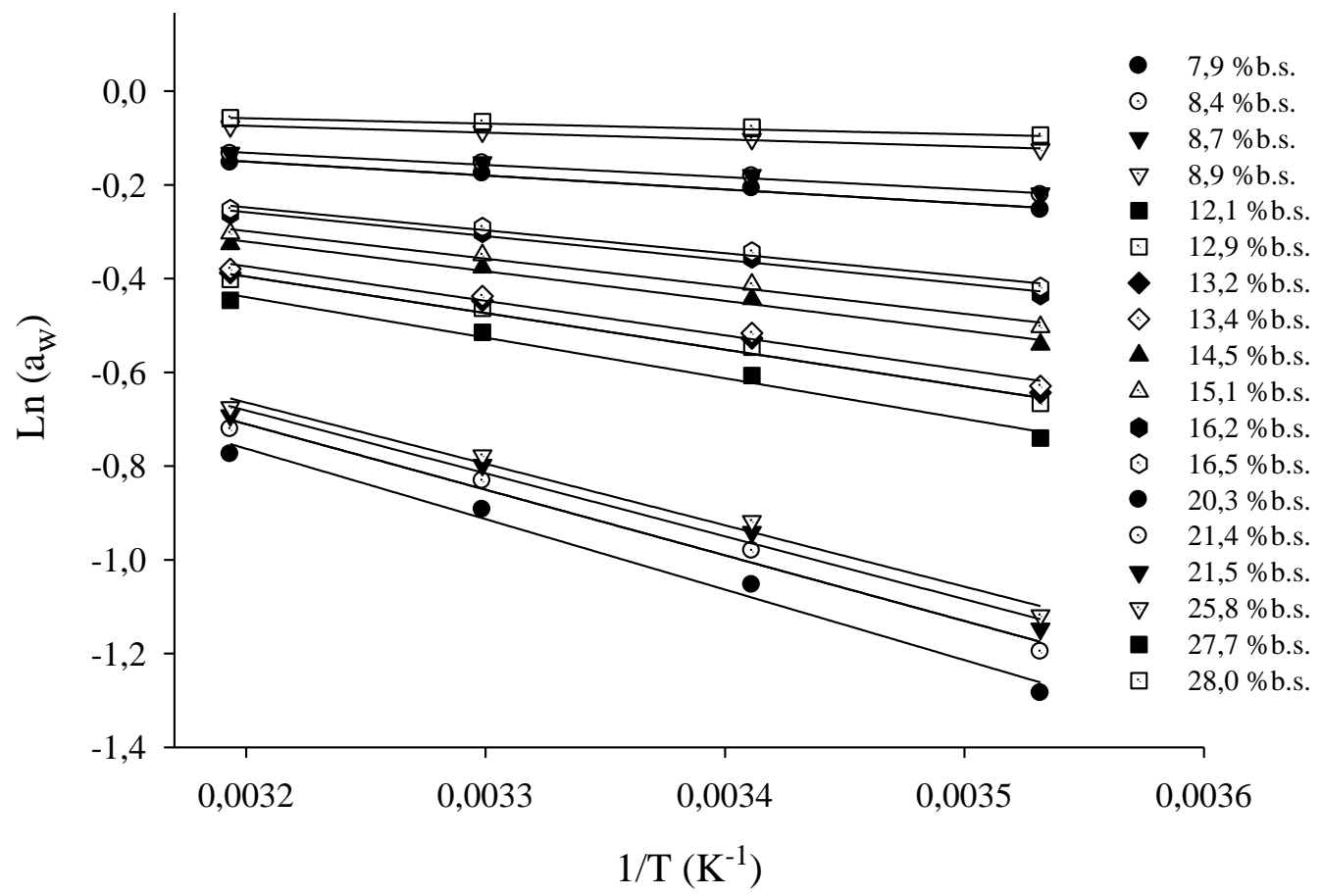

FIGURA 2. Curvas do logaritmo neperiano da atividade de água (decimal), para o teor de água de equilíbrio (\% b.s.), em função de valores de $1 / \mathrm{T}\left(\mathrm{K}^{-1}\right)$ para as sementes de sorgosacarino. Curves of the logarithm of the wate $r$ activity (decimal) for hygroscopic equilibrium (\% d.b.), according to values of $1 / \mathrm{T}\left(\mathrm{K}^{-1}\right)$ for $s$ weet sorghum seeds.

Na Figura 3, está apresentada a curva do calor isostérico integral de dessorção, em função do teor de água de equilíbrio (\% b.s.), para as sementes de sorgo-sacarino.

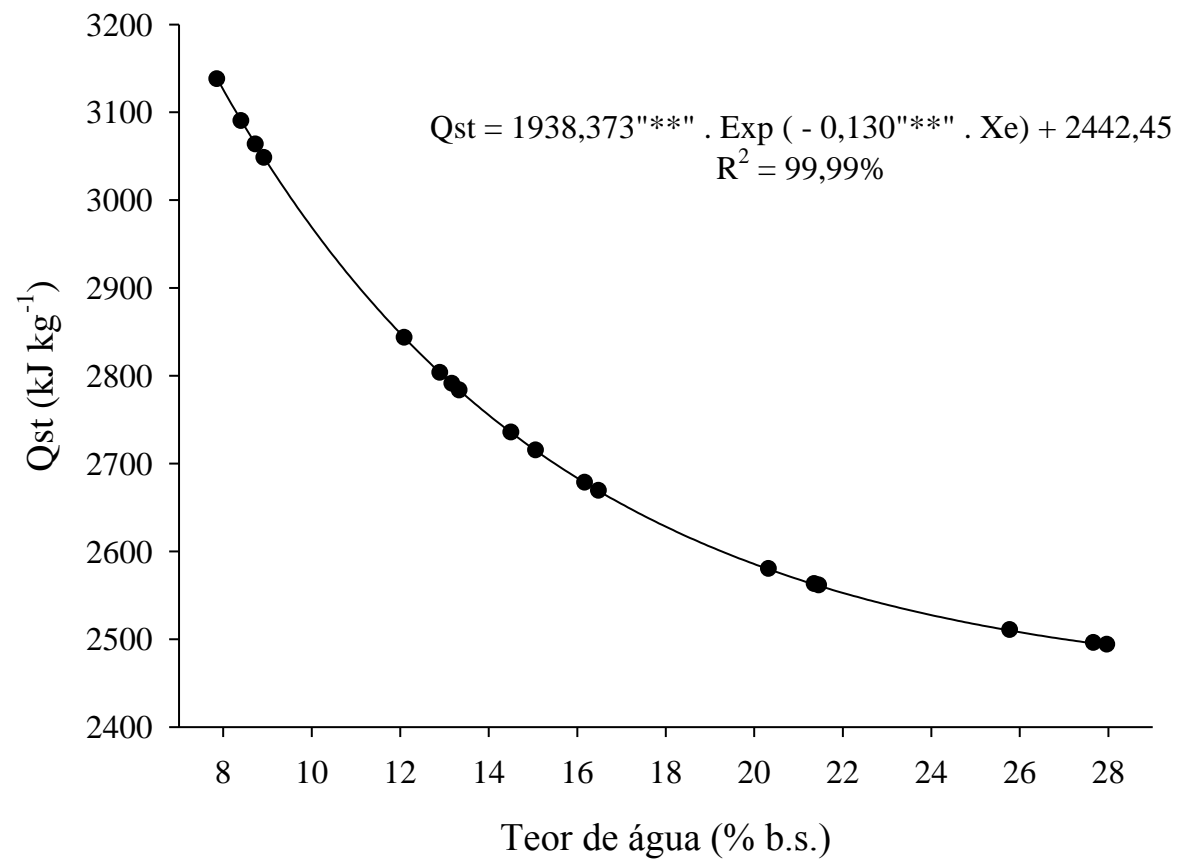

*** Significativo a $5 \%$, pelo teste $\mathrm{t}$

FIGURA 3. Valores experimentais e estimados do calor isostérico integral de dessorção $\left(\mathrm{Q}_{\mathrm{st}}\right)$ das sementes de sorgo-sacarino em função do teor de água de equilíbrio. Experimental and estimated values of the isosteric heat of desorption $\left(Q_{s t}\right)$ for sweet sorghum seeds as a function of hygroscopic equilibrium. 
Observa-se, na Figura 3, que os valores de calor isostérico integral de dessorção para as sementes de sorgo-sacarino, entre os teores de água de equilíbrio de 7,9 e 24,0\% (b.s.), variaram de $3.136,54$ a $2.493,33 \mathrm{~kJ} \cdot \mathrm{kg}^{-1}$.

Verificou-se que, com a redução do teor de água, ocorreu o aumento da energia necessária para a remoção de água do produto representada pelos valores do calor isostérico integral de dessorção, conforme relatado para diversos produtos agrícolas, como crambe (COSTA et al., 2013), pimenta-malagueta (SILVA \& RODOVALHO, 2012) e pinhão (OLIVEIRA, 2010).

COSTA et al. (2013) encontram magnitudes compreendidas entre 10.183,33 e 3.538,74 kJ.kg${ }^{1}$, trabalhando com crambe para o teor de água de equilíbrio entre 1,22 e 3,39\% (b.s.). Já AYRANCI \& DUMAN (2005) obtiveram valores de calor isostérico semelhantes aos obtidos neste trabalho,

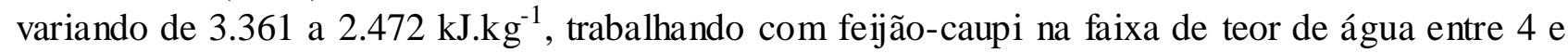
$16 \%$ (b.s.).

OLIVEIRA et al. (2013) relataram que os valores do calor isostérico para os grãos de milho variaram de $2.506 \mathrm{~kJ} \mathrm{~kg}^{-1}$ a $2.734 \mathrm{~kJ} \mathrm{~kg}^{-1}$, para a faixa de teor de água de $23,3 \%$ a $12,8 \%$ (b.s.).

WANG \& BRENAN (1991) relatam ainda que, à medida que as moléculas de água vão ligando-se quimicamente aos sítios de sorção ativos, a sorção passa a ocorrer em sítios menos ativos em que estão com elevado teor de água, com menor energia de interação e, consequentemente, com menor calor isostérico de sorção.

O calor isostérico integral de dessorção para as sementes de sorgo-sacarino pode ser representado de forma adequada pela equação de regressão exponencial, a qual apresentou elevado coeficiente de determinação $(99,9 \%)$, como também foi constatado por COSTA et al. (2013) e CAETANO et al. (2012).

\section{CONCLUSÕES}

1. O teor de água de equilíbrio das sementes de sorgo diminui com o aumento da temperatura, para uma mesma atividade de água.

2. As isotermas de dessorção obtidas para as sementes de sorgo-sacarino possuem formato sigmoidal, padrão para o equilíbrio higroscópico de produtos agrícolas amiláceos.

3. O modelo de Chung-pfost é o que melhor representa a higroscopicidade das sementes de sorgo-sacarino.

4. O calor isostérico integral de dessorção das sementes de sorgo-sacarino varia de 3.136,54 a $2.493,33 \mathrm{~kJ} . \mathrm{kg}^{-1}$, para a faixa de teor de água de 7,9 a 28,0 (\% b.s.).

\section{REFERÊNCIAS}

AGRICULTURAL ENGINEERS YEARBOOK OF STANDARDS. St. Joseph: American Society of Agricultural Engineers, 2003.

AYRANCI, E.; DUMAN, O. Moisture sorption isotherms of cowpea (Vigna Unguiculata L. Walp) and its protein isotate at 10, 20 and $30^{\circ} \mathrm{C}$. Journal of Food Engineering, Davis, v. 70, n. 1, p. 83-91, 2005.

BRASIL. Ministério Da Agricultura e Reforma Agrária. Secretaria nacional de defesa agropecuária. Regras para Análise de Sementes. Brasília, 2009. 398 p.

CAETANO, G. C.; SOUSA, K. A.; RESENDE, O.; SALES, J. F. COSTA, L. M. Higroscopicidade de sementes de caju-de-árvore-do-cerrado. Pesquisa Agropecuária Tropical, Goiânia, v. 42, n. 4, p. 437 445, out./dez. 2012.

CAVALCANTI MATA, M. E. R. M; DUARTE, M. E. M.; FIGUEIREDO, R. M. F. Obtenção de frutos em pó. Campina Grande: Universidade Federal de Campina Grande, 2003. 15p. Projeto CNPq. 
CHEN, C.; WENG, Y. Moisture sorption isotherms of Oolong Tea. Food Bioprocess Technology, New York, v.3, n.2, p.226-233, 2010.

CHINNAN, M. S.; BEAUCHAT, L. R. Sorption isotherms of whole cowpeas and flours. In: Lebensm - Wiss u. Tech., [s.n.], v. 18, p. 83-88, 1985.

CHUNG, D.S.; PFOST, H.B. Adsorption and desorption of water vapors by cereal grains and their products Part II. Transactions of the ASAE, St. Joseph, v.10, p. 549-551, 1967.

CORRÊA, P. C.; MARTINS, D. S. R.; MELO, E. C. Umigrãos: programa para o cálculo do teor de umidade de equilíbrio para os principais produtos agrícolas. Viçosa: Centreinar - UFV, 1995. 10 p.

COSTA, L.M.; RESENDE, O.; OLIVEIRA, D.E.C. Isotermas de dessorção e calor isostérico dos frutos de crambe. Revista Brasileira de Engenharia Agrícola e Ambiental, Campina Grande, v.17, n.4, p.412-418, 2013.

DALPASQUALE, V. A.; SPERANDIO, D. Modelo de simulação de secagem de produtos agrícolas usando entalpia do ar constante. Engenharia Agrícola, Jaboticabal, v.30, n.4, p. 726-731, 2010.

DRAPER, N. R.; SMITH, H. Applied regression analysis. 3 ed. New York: John Wiley and Sons, 1998. 712p.

FARAHNAKY, A.; ANSARI, S.; MAJZOOBI, M. Effect of glycerol on the moisture sorption isotherms of figs. Journal of Food Engineering, New York, v.93, n.4 , p.468-473, 2009.

GONELI, A. L. D.; CORRÊA, P.C.; OLIVEIRA, G.H.H.; BOTELHO, F.M. Water desorption and thermodynamic properties of okra seeds. Transactions of the Asae, St. Joseph, v. 53, n. 1, p. 191197, 2010.

HALSEY, G. Physical adsorption on uniform surfaces. The Journal of Che mical Physics, New York, v. 16, n. 10, p. 931-937, 1948.

HENAO, J. D.; QUEIROZ, M. R.; HAJ-ISA, N. M. A. Umidade de equilíbrio de café cereja descascado baseada em métodos estático e dinâmico. Revista Brasileira de Engenharia Agrícola e Ambiental, Campina Grande, v.13, n.4, p.470-476, 2009.

HENDERSON, S. M. A basic concept f equilibrium moisture content. Agricultural Engineering, St. Joseph, v. 33, p. 29-31, 1952.

HUBINGER, M. D.; VIVANCOPEZANTES, D.; KUROZAWA, L. E.; SOBRAL, P. J. A. Isotermas de dessorção de filé de bonito (Sarda Sarda) desidratado os moticamente e defumado.

Revista Brasileira de Engenharia Agrícola e Ambiental, Campina Grande, v.13, n.3, p.305-311, 2009.

IGLESIAS, H.; CHIRIFE, J. Prediction of the effect of temperature on water sorption isotherms of food material. Journal of Food Technology, Oxford, v. 11, n.2, p.109-116, 1976.

IGUAZ, A.; VÍRSEDA, P. Moisture desorption isotherms of roung rice at high temperatures. Journal of Food Engineering, Londres, v. 79, n. 3, p. 794-802, 2007.

LIMA, E. E. DE; SILVA, A. S.; FIGUEIREDO, R. M. DE F.; QUEROZ, A.J. DE M. Estudo das isotermas e calor isostérico de adsorção da farinha do coroa de frade. Revista Brasileira de Produtos Agroindustriais, Campina Grande, v.10, n.2, p.163-170, 2008.

MADAMBA, P. S.; DRISCOLL, R. H.; BUCKLE, K. A. The thin layer drying characteristic of garlic slices. Journal of Food Engineering, Essex, v. 29, n. 1, p. 75-97, 1996.

OLIVEIRA, F. Adsorption isotherms of pinhão (Araucaria angustifolia seeds) starch and thermodynamic analysis. Journal of Food Engineering, Essex, v.100, n.3, p. 468-473, 2010.

OLIVEIRA D.E.C.; RESENDE, O.; SMANIOTTO, T.A.S.; SOUSA, K.A.; CAMPOS, R.C. Propriedades termodinâmicas de grãos de milho para diferentes teores de água de equilíbrio. Pesquisa Agropecuá ria Tropical, Goiânia, v. 43, n. 1, p. 50-56, 2013. 
PARK, K. J.; NOGUEIRA, R. I. Modelo para ajuste de isoterma de sorção de alimentos. Revista Engenharia Rural, Piracicaba. 3, v. 1, p. 80-86, 1992.

RESENDE, O.; ULLMANN, R.; SIQUEIRA, V.C.; CHAVES, T.H.; FERREIRA, L.U. Modelagem matemática e difusividade efetiva das sementes de pinhão-manso (Jatropha curcas L.) durante a secagem. Engenharia Agrícola, Jaboticabal, v.31, n.6, p.1123-1135, 2011.

SANTOS, S. B.; SAMPAIO, C. P.; MARTINS, M. A.; ARAÚJO, R. F. Isotermas de equilíbrio higroscópico para grãos de pinhão manso (Jatropha curcas L.). Revista Brasileira de Armazenamento, Viçosa, MG, v. 37, n. 1-2, p. 48-53. 2013.

SILVA, H. W.; RODOVALHO, R. S. Isotermas de dessorção das sementes de pimenta malagueta. Global Science and Technology, Rio Verde, v. 05, n. 01, p.32 - 39, jan/abr. 2012.

SMANiOTTO, T. A. S.; RESENDE, O.; OLIVEIRA, D. E. C.; SOUS A, K. A.; CAMPOS, R.C. Isotermas e calor latente de dessorção dos grãos de milho da cultivar ag 7088. Revista Brasileira de Milho e Sorgo, Sete Lagoas, v.11, n.3, p. 312-322, 2012.

SOUSA, K. A.; RESENDE, O; COSTA, L. M. Isotermas de des sorção das sementes de nabo forrageiro obtidas pelos métodos dinâmico e estático. Revista Brasileira de Engenharia Agrícola e Ambiental, Campina Grande, v.17, n.2, p.216-222, 2013.

TEIXEIRA, C. G.; JARDINE, J. G.; BEISMAN, D. A. Utilização do sorgo sacarino como matéria prima complementar à cana-de-açúcar para obtenção de etanol em microdestilaria. Ciência e Tecnologia de Alime ntos, Campinas, v. 17, n.3, p. 221- 229, 1997.

ULLMANN, R.; RESENDE, O.; SALES, J. F.; CHAVES, T. H. Qualidade das sementes de pinhão manso submetidas à secagem artificial. Revista Ciência Agronômica, Fortaleza, v. 41, n. 03, p. 442-447, 2010.

VAN DER BERG, C. Description of water activity of foods for engineering purposes by means of the GAB model of sorption. In: MCKENNA, B. M. Engineering and food. London: Else vier Applied Science, 1984,v. 1, p.311-321.

WANG, N.; BRENNAN, J. G. Moisture sorption isotherm characteristics of potato at four temperatures. Journal of Food Engineering, New York, v.14, n.1, p.269-287, 1991. 